\title{
CELLULAR MESOBLASTIC NEPHROMA: REPORT OF 3 CASES
}

\author{
M. Ramani, C. Sandhya Rani, K. Geetha, K. Ramesh Reddy.
}
1. Professor, Department. of Pathology, Niloufer Hospital for Women and Children.
2. Post Graduate, Department of Pathology, Niloufer Hospital for Women and Children
3. Assistant Professor, Department of Pathology, Niloufer Hospital for Women and Children
4. Professor, Department of Pediatric Surgery, Niloufer Hospital for Women and Children

\section{CORRESPONDING AUTHOR:}

Dr Ramani M,

Professor, Department of Pathology,

Osmania Medical College,

Koti, Hyderabad, Andhra Pradesh, India.

E-mail: drmramani@sify.com

\begin{abstract}
Mesoblastic nephroma is a relatively rare infantile renal tumor. It comprises 3-6\% of pediatric renal masses. It comprises of three histological types namely classic, cellular and mixed variants. Cellular variant accounts for $60 \%$ cases and is associated with poor prognosis with survival rate of $85 \%$ compared to $100 \%$ for classic variant. It remains a diagnostic challenge for pathologists due to its similarity to other more frequent kidney neoplasms. Here we report 3 cases of cellular mesoblastic nephroma which presented over a period of 4 years from January 2007 to December 2010.
\end{abstract}

KEYWORDS: cellular mesoblastic nephroma, poor prognosis, differential diagnosis.

INTRODUCTION: Mesoblastic nephroma first described by Bolande et al. in 1967 as leiomyoma like tumor of kidney ${ }^{1}$. It's a congenital neoplasm commonly diagnosed before 6 months age. Histological variants include classic, cellular and mixed. Cellular variant demonstrates more local recurrences and invasion of perirenal fat, adjacent organs, distant metastasis, and accompanied by intramural hemorrhage with large cystic or necrotic component. Most renal neoplasms in childhood are represented by Wilms tumor, leading to treatment directed at Wilms tumor, without pathological confirmation. Renal neoplasms in neonates younger than 6 months are rare. Other neoplasms which occur in this age group include mesoblastic nephroma, multi cystic nephroma, rhabdoid tumor, clear cell sarcoma, Ewing's sarcoma, PNET, desmoplastic small round cell tumor, meta nephric stromal tumor, poly phenotypic anaplastic sarcoma, ossifying renal tumor of infancy, embryonal sarcoma, intra renal teratoma, epitheloid tumor, intra renal pure yolk sac tumor. Among these, mesoblastic nephroma is frequent one, with $90 \%$ cases being diagnosed within first year of life. It is treated by nephrectomy. Bandyopadhyay et al suggests that its diagnosis remains a challenge for pathologists, due to its similarities with pediatric renal neoplasms. The lack of familiarity with this entity leads to misdiagnosis².

MATERIALS \&METHODS: We report 3 cases of cellular mesoblastic nephroma from Niloufer Hospital, Hyderabad, India over a period of four years. Nephrectomy was done in all 3 cases and sent for histopathological examination. Haemotoxylin and eosin stained sections examined. Immuno histochemistry performed with vimentin. 


\section{CASE REPORT}

\section{RESULTS:}

CASE 1: 4 month old female child brought by parents with complaints of lump abdomen since 20 days, gradually increasing in size, refusal of feeds, clinical examination revealed Abdominal distension, non tender, firm lump in right loin, no hepatosplenomegaly. Baseline investigations revealed mild anemia, mild elevation of blood urea and serum creatinine. Ultrasound examination \& CT scan were suggestive of WILMS TUMOR RIGHT KIDNEY. Right nephrectomy, biopsy from ileo ceacal region performed with suspicion of tumor invasion and sent for HPE.

GROSS EXAMINATION: We received right uretero nephrectomy specimen measuring $8 \times 7 \times 5 \mathrm{cms}$, ureter measuring $4 \mathrm{cms}$ in length. Surface smooth, firm in consistency; cut section showed grey white tumor tissue with whorled appearance, areas of hemorrhage, multiple cystic areas, adjacent normal appearing kidney. Also received bowel segment measured $2.5 \times 0.5 \mathrm{~cm}$ looking like appendix with ileo-ceacal area.

CASE 2: 11 month old male child brought with complaints of mass in right side of abdomen since 10 days gradually increasing in size. On examination, mass measuring $12 \times 14 \mathrm{~cm}$ noted extending, from right hypochondrium to right iliac fossa not moving with respiration. All borders well defined, fullness noted in right renal angle, suggesting it to be a renal mass. Base line investigations were normal. Ultra sound examination and CT scan were suggestive of WILMS TUMOR RIGHT KIDNEY. Right nephrectomy performed. During surgery, no lymph nodes no infiltration to adjacent structures noted.

GROSS EXAMINATION: We received nephrectomy specimen measuring $13 \times 6 \times 3 \mathrm{~cm}$ (figure 1 ), ureter not identified had smooth surface and firm consistency. Cut section grey white tumor tissue in the lower pole with adjacent normal appearing kidney tissue.

CASE 3: 10 day old female child brought with complains of mass abdomen, abdominal distension since 3 days. On examination lump of size $7 \times 5 \mathrm{~cm}$ was noted in left loin. Ultra sound examination suggested intra abdominal mass measuring $8 \times 6 \mathrm{~cm}$, diagnosis of DERMOID CYST made. During surgery tumor noted to arise from left kidney and left nephrectomy performed.

GROSS EXAMINATION: We received left nephrectomy specimen measuring $9 \times 6.5 \times 5 \mathrm{~cm}$, with attached ureter measuring $3 \mathrm{cms}$. Cut section revealed grey white tumor tissue with normal appearing kidney tissue.

MICROSCOPIC EXAMINATION: Histopathological examination of sections stained by haematoxylin and eosin in three cases revealed tumor tissue arranged in interlacing bundles (figure 2), diffuse sheets with pushing borders with adjacent normal appearing kidney tissue. The individual cells were spindle in shape with scant cytoplasm, elongated nucleus, with prominent nucleolus. With mitotic activity of 3-4/10hpf. Additionally case 1 showed areas of hemorrhage and necrosis, small micro cystic areas (figure 3), biopsy from iliocaecal area revealed extension of tumor tissue up to sub mucosa. 


\section{CASE REPORT}

IMMUNOHISTOCHEMISTRY: Immuno histochemistry with vimentin showed strong positivity in all three cases.

DISCUSSION: Mesoblastic nephroma is benign hamartomatous mal-development of fetal kidney. It was first described by Bolande et al in 19671. Mesoblastic nephroma also known as fetal, mesenchymal or leiomyomatous hamartoma. Usually discovered before 6 months of age. Mesoblastic nephroma presents as an asymptomatic abdominal mass, sometimes accompanied by hematuria. In most cases, the diagnosis is made in the neonatal period, as it leads to polyhydramnios (71\% of the gestations associated with tumor), hydrops and premature birth, hypertension (as a result of increase in renin levels by renal infiltration) and hypercalcaemia (due to tumor's secretion of a substance similar to parathormone) ${ }^{3,4}$.

Grossly, tumor is solid, with yellowish gray tan, with whorled configuration reminiscent of uterine leiomyoma. Most are centered near hilus of kidney. The tumor is usually well circumscribed, but may infiltrate renal parenchyma and perirenal fat. Areas of hemorrhage and necrosis are usually absent. A cystic variant of tumor has been described ${ }^{5,6}$.

Microscopically, classic variant shows a variable cellular growth of spindle cells. Wigger ${ }^{7}$ believes that these cells have features of secondary mesenchyme. The proliferating cells acquire features of fibroblasts, myofibroblasts, or smooth muscle cells ${ }^{8}$, 9. Tubules and glomeruli may be seen surrounded by spindle tumor cells. Small islands of hyaline cartilage, foci of extramedullary hematopoiesis may be present. There is no capsule separating tumor from uninvolved renal parenchyma. Cellularity, mitotic activity is high in cellular variant and have tendency to infiltrate renal pelvis, perirenal tissue, adjacent structures, may contain areas of hemorrhage necrosis ${ }^{10}$ and tends to be more aggressive. When classic and cellular patterns coexist, tumors are designated as mixed. Three variants are immunoreactive to vimentin, smooth-muscle actin, and desmin, negative for epithelial markers ${ }^{11}$.

It has been suggested ${ }^{12}$ that tumor arises from secondary mesenchyme which is no longer capable of producing epithelial cells. Increased ${ }^{13}$ chromosome 11 copy numbers is a characteristic aberration in cellular mesoblastic nephroma. Additional characteristic polysomies in cellular or mixed histology include extra copies of chromosomes 8 and 17. In contrast, chromosome 11 aberrations were not found in classic mesoblastic nephroma. These genetic aberrations described do not seem to correlate with prognosis ${ }^{13}$. Recent studies ${ }^{14}$ have shown classic and cellular variants have additional genetic differences. Only cellular variant shows translocation $(12 ; 15)$ (p13; q25), which leads to ETV6-NTRK3 gene fusion. Identical translocation has also been reported in infantile fibro sarcoma, suggesting that cellular subtype $\mathrm{CMN}$ would represent an intrarenal infantile fibro sarcoma ${ }^{15}$.

When fusiform cells predominate, differential diagnosis must include Wilms tumor, clearcell type RCC (fusiform cell pattern), and clear cell sarcoma. Heterologous differentiation, blastemal elements, nephrogenic rests favor diagnosis of Wilms tumor. Interlacing pattern of fascicles, extension of fascicles into adjacent renal parenchyma, stag horn vessels, coarse nuclear chromatin, and absence of clear cell pattern, desmin \&muscle specific actin positivity differentiates it from clear cell sarcoma.

Mesoblastic nephroma is treated with radical nephrectomy to reduce the risks of local recurrence. This treatment usually enough, as tumor has low malignancy potential ${ }^{16}$. Only $5 \%$ of 
patients have recurrence, usually within first year after nephrectomy and most of them are cellular subtype. Therefore, during first year after nephrectomy, patient must be submitted to series of ultrasonographic assessments, in order to detect early signs of local recurrence ${ }^{3,4}$. Cellular variant is associated with worse prognosis than classic variant. Metastatic disease, to lungs, liver, brain, heart and extensive area of local recurrence are rare events. Risk factors ${ }^{1}$ for recurrence are positive surgical margins, tumor rupture during resection, cellular subtype and patient's age (older than 3 months). The ideal therapy to control recurrence is yet to be clarified.

CONCLUSION: In conclusion we suggest that mesoblastic nephroma should be considered as differential diagnosis in pediatric renal neoplasms, especially in early neonatal period and should be excluded from other tumors in infancy by intraoperative cytological examination, histopathological examination, and immuno histochemistry. Because this alters course of treatment, further surveillance and prognosis of patient.

\section{REFERENCES:}

1. Bolende RP, Brough AJ, Izant RJ: Congenital mesoblastic nephroma of infancy: Pediatrics; 1967; 40: 272.

2. Bandyopadhyay R, Chatterjee U, Mondal SK, Banerjee S, Chatterjee US. Unusual morphology in mesoblastic nephroma. Pediatric Surg Int 2009; 25:109-12

3. Sebire NJ, Vujanic GM. Paediatric renal tumours: recent developments, new entities and pathological features. Histopathology 2009; 54:516-28

4. Glick RD, Hicks MJ, Nuchtern JG, Wesson DE, Olutoye 00, Cass DL. Renal tumors in infants less than 6 months of age. J Pediatr Surg 2004; 39:522-5.

5. Drut R: Multicystic congenital mesoblastic nephroma. Int J Surg Pathol 2002; 10:59-63.

6. Ganick DJ, Gilbert EF, Beckwith JB, Kiviat N: Congenital cystic mesoblastic nephroma. Hum Pathol 1981; 12:1039-1043

7. Wigger HG: Fetal mesenchymal hamartoma of kidney. A tumor of secondary mesenchyme. Cancer 1975; 36:1002-1008.

8. Bogdan R, Taylor DEM, Mostofi FK: Leiomyomatous hamartoma of the kidney. A clinical and pathologic analysis of 20 cases from the Kidney Tumor Registry. Cancer 1973; 31:462-467.

9. Shen SC, Yunis EJ: A study of the cellularity and ultrastructure of congenital mesoblastic nephroma. Cancer 1980; 45:306-314.

10. Sandstedt B, Delemarre JFM, Krul EJ, Tournade MF: Mesoblastic nephromas. A study of 29 tumours from the SIOP nephroblastoma file. Histopathology 1985; 9:741-750.

11. Kumar S, Marsden HB, Carr T, Kodet R: Mesoblastic nephroma contains fibronectin but lacks laminin. J Clin Pathol 1985; 38:507-511.

12. H B marsden andWA newton Jnrt J Clin Pathol. 1986 May; 39(5): 508-513

13. Deborah E. Schofield,t Eduardo J. Yunis, and Jonathan A. Fletcher. Chromosome Aberrations in Mesoblastic Nephroma: American Journal of Pathology, Vol. 143, No. 3, September 1993

14. Argani P, Fritsch M, Kadkol S, Schuster A, Beckwith JB, Perlman EJ: Detection of the ETV6NTRK3 chimeric RNA of infantile fibrosarcoma/cellular congenital mesoblastic nephroma in 


\section{CASE REPORT}

paraffin-embedded tissue: application to challenging pediatric renal stromal tumors. Mod Pathol 2000; 13:29-36.

15. Rubin BP, Chen C-J, Morgan TW, Xiao S, Grier H, Kozakewich HP, Perez-Atayde AR, Fletcher JA: Congenital mesoblastic nephroma $\mathrm{t}(12 ; 15)$ is associated with ETV6-NTRK3 gene fusion. Cytogenetic and molecular relationship to congenital (infantile) fibrosarcoma. Am J Pathol 1998; 153:1451-1458.

16. Lina Gomes dos Santos; Juliana de Sousa Ribeiro de Carvalho; Marcela Aguiar Reis; Rayli Lauro Jennyfer Brandão Sales Cellular congenital mesoblastic nephroma: case report J. Bras. Nefrol. vol.33 no.1 São Paulo Jan./Mar. 2011
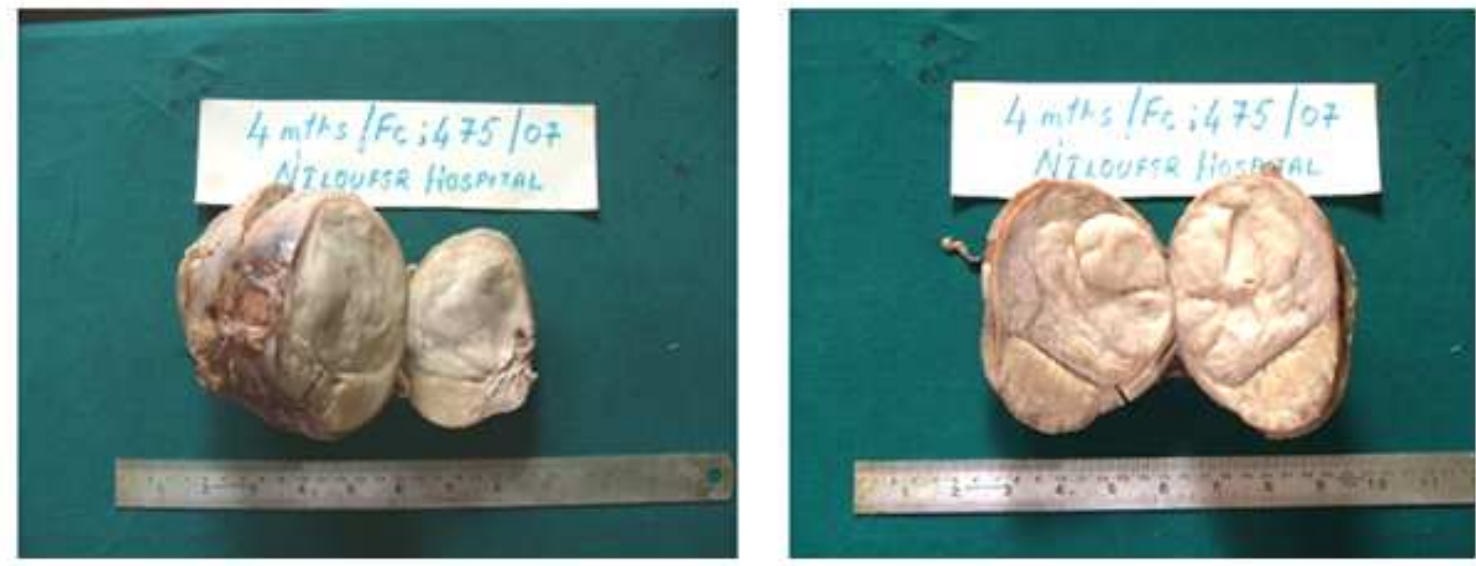

Figure1: Gross Cut Section showing a grey /white tumor, with whorled appearance adjacent normal kidney
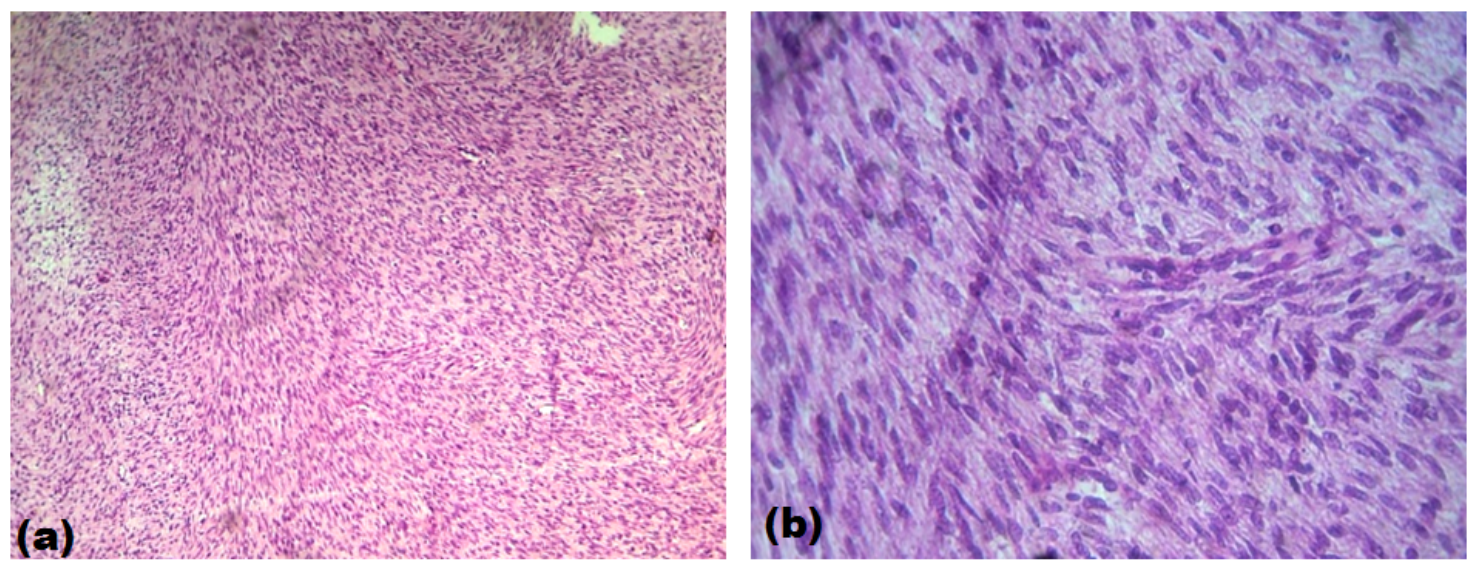

Figure 2: (a) H\&E(10X)-Densely packed spindle cells arranged in fascicles and bundles. (b) H\&E(40X)-Plump spindle cells with moderate amount of cytoplasm, cigar shaped nucleus and prominent nucleoli 


\section{CASE REPORT}

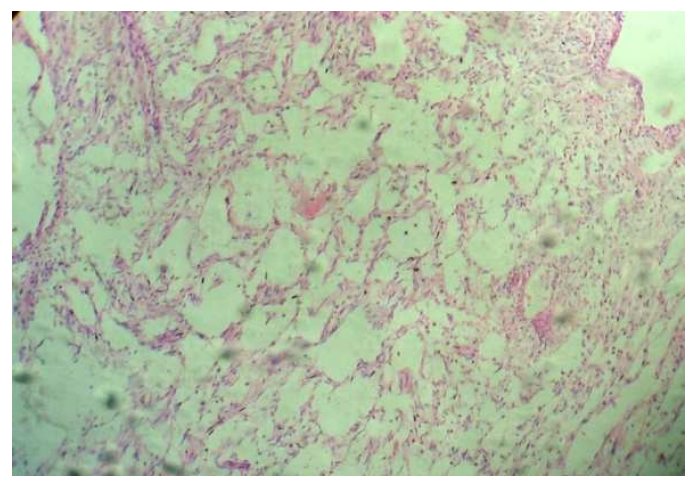

Figure 3: H\&E(40X) showing microcystic areas

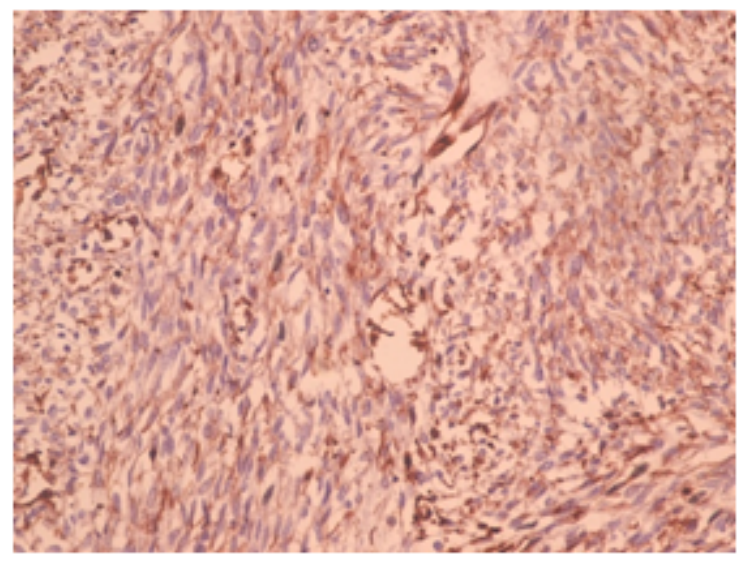

Figure4: IHC(40X) vimentin showing positivity for vimentin 